\title{
The Correlation between the CT Liver Volume and the Actual Weight of the Resected Cirrhotic Liver in Transplantation Patients
}

\author{
Kulyada Eurboonyanun, M.D. ${ }^{1}$, Chalerm Eurboonyanun, M.D. ${ }^{2}$, Julaluck Promsorn, M.D. , \\ Jiranthanin Phaorod, M.D. ${ }^{1}$, Tharatip Srisuk, M.D. ${ }^{2}$, Nittaya Chamadol, M.D. ${ }^{1}$, \\ Mukesh Harisinghani, M.D. ${ }^{3}$
}

${ }^{1}$ Department of Radiology, Faculty of Medicine, Khon Kaen University, Mueang, Khon Kaen 40002, Thailand. ${ }^{2}$ Department of Surgery, Faculty of Medicine, Khon Kaen University, Mueang, Khon Kaen 40002, Thailand.

${ }^{3}$ Abdominal Imaging Division, Radiology Department, Massachusetts General Hospital, Boston, United States of America. Received 1 July 2021 • Revised 17 September 2021 • Accepted 28 September 2021 • Published online 29 November 2021

\begin{abstract}
:
Objective: Volumetric assessment with computed tomography (CT), known as CT volumetry, is the preferred method for estimating future liver remnant. However, the data regarding the usage of CT volumetry to estimate future liver remnant of the diseased liver is still lacking. This study was designed to evaluate the correlation between the liver volume, calculated by CT, and the actual weight of the resected liver in patients who underwent orthotopic liver transplantation. Material and Methods: A total of 32 patients having underwent liver transplantation; from March 2009 to June 2015, were included. A radiologist retrospectively reviewed the pre-operative CT and performed the volume measurement. Statistical analysis was performed to determine the relationship between the estimated liver volume and the actual liver weight.

Results: The estimated liver volume was significantly different among the cirrhosis of different etiology ( $p$-value=0.001 for the total liver volume and $p$-value $=0.003$ for the functional liver volume). Compared with the total liver volume, the functional liver volume had a stronger correlation with the actual weight of the resected liver $(r=0.955$ vs. $r=0.786)$. The following formula can be used to accurately estimate the expected weight of the resected liver (expected liver weight: ELW), based on the estimated functional liver volume (FLV) derived by CT volumetry: ELW=489.531+(0.618*FLV). The R-squared for this regression model was 0.914 .
\end{abstract}

Contact: Chalerm Eurboonyanun, M.D.

Department of Surgery, Faculty of Medicine, Khon Kaen University,

Mueang, Khon Kaen 40002, Thailand.

E-mail: chaleu@kku.ac.th

() 2021 JHSMR. Hosting by Prince of Songkla University. All rights reserved.

This is an open access article under the CC BY-NC-ND license

(http://www.jhsmr.org/index.php/jhsmr/about/editorialPolicies\#openAccessPolicy).
J Health Sci Med Res 2022;40(4):437-447 doi: 10.31584 /jhsmr.2021855 www.jhsmr.org 
Conclusion: CT volumetry is reliable and accurate in predicting the actual amount of the resected liver parenchyma in cirrhotic patients.

Keywords: computed tomography volumetry, hepatic resection, liver surgery, liver transplantation, liver volume

\section{Introduction}

Liver resection is the treatment of choice in patients with curative primary hepatic malignancies and liver metastasis from certain primary tumors. ${ }^{1-7}$ This surgery is also performed on the living donor for liver transplantation. Regardless of the purpose, liver resection is a major and complex operation, and comes with risks of complications. Post-hepatectomy liver failure, one of the most devastating complications of partial liver resection, can be found in up to $35.0 \%$ of patients. It is associated with a high mortality rate of up to $59.0 \%{ }^{8,9}$ Many risk factors are related to post-hepatectomy liver failure, and future liver remnant (FLR) is one of the most recognized. If the liver remnant is too small, the intra-sinusoidal pressure will increase, leading to severe hepatocyte damage. In general, FLR of $<20.0 \%$ in healthy livers, FLR of $<30.0 \%$ in non-cirrhotic, post-hepatotoxic chemotherapy livers, and FLR of $<40.0-$ $50.0 \%$ in fatty- or cirrhotic livers are considered risk factors for post-hepatectomy liver failure. Thus, it is imperative to maintain an adequate volume of residual liver parenchyma to ensure the proper functionality of the liver after hepatic resection. ${ }^{8-11}$ For surgical-candidate patients with low estimated FLR, radiological or surgical interventions may be performed to pre-operatively increase FLR, and alleviate the risk of post-hepatectomy liver failure. An accurate mean of follow-up of liver volume after these procedures is essential in the treatment planning for such patients. ${ }^{12-14}$

There are two methods for measuring the amount of resected liver intra-operatively: liver weight measurement and liver volume measurement. The volume measurement is usually performed by immersing the resected liver in a water bath filled with normal saline solution, and measuring the volume of the displaced liver (Archimedes' principle). However, this method is less practical than the liver weight measurement. $^{15}$

Currently, there are several means for the estimation of FLR. One approach calculates the FLR from the formula comprising of variable clinical measurements; such as, body weight, height and surface area. However, the outcomes of most formulas are varied and relatively inaccurate. ${ }^{16-18}$ On the other hand, the imaging-based approach, specifically computed tomography (CT), is accurate and correlates well with the actual liver volume and weight. Many methods have been proposed to convert CT liver volume to the resected liver's weight and volume. ${ }^{16,19-22}$

Because, the amount of FLR required for the resection of a diseased liver is considerably larger than the amount required for the resection of a healthy liver, a small resection might lead to major complications. Thus, an accurate pre-operative estimation of the liver volume is crucial in this group of patients. ${ }^{14,23}$ To date, most of the data regarding the accuracy of CT volumetry in the liver volume assessment has come from studies that used normal livers from living donors as the reference. However, in cirrhotic livers, the inflammation of the liver, due to various etiologies, leads to the deposition of fibrosis. It is unclear whether this histopathological change affects the weight of the liver. ${ }^{24}$ By assuming that the weight of a cirrhotic liver would be similar to a healthy liver, one might over-or underestimate the amount of the functioning liver.

The accuracy of CT volumetry in diseased livers has not yet been widely validated, because the studies are 
scarce. For each patient planned for partial liver resection, the surgical planning could be substantially different; ranging from non-anatomical resection to extended lobectomy to trisectionectomy. Therefore, the pre-operative volume estimation requires a multidisciplinary assessment which is carefully tailored for each patient. Thus, retrospective CT volume estimation without such inspection might lead to a wrong assumption. On the contrary, using the whole explanted liver as a reference allows a more direct comparison without such confounders. The objective of this study was to assess the correlation between the volume estimated by pre-operative CT and the actual weight of the resected diseased liver of patients who underwent whole liver transplantation.

\section{Material and Methods}

This retrospective study was reviewed and approved by the Institutional Ethics Committee for Human Research under the Helsinki Declaration and the $\mathrm{ICH}$ Good Clinical Practice Guidelines.

All patients who underwent whole liver transplantation from March 2009 to June 2015 were included in this study. The exclusion criteria were: (1) no pre-operative CT available in the Picture Archiving and Communication Systems, (2) no record of the resected liver weight on the operative note, and (3) no operative note stored in the electronic medical records. If a single patient underwent multiple transplantations, only the first session would be included.

Demographic and clinical data comprising of age, gender, liver pathology, Child-Pugh classes, indication for liver transplantation, and resected liver weight were obtained from electronic medical records. During the operation, after the recipient's liver was removed, the vessels of the resected liver would be de-clamped to release the blood retained in the lumen. The organ was weighed on the back table, using a digital scale.
Pre-operative CT was performed using three multidetector computed tomography machines (Philips Brilliance ICT SP-128 slices, Siemens Somatom Definition Flash-128 slices, and Siemens Somatom Plus-4 slices). Most patients underwent a routine dual-phase CT protocol that included non-contrast CT, late-arterial-phase CT (30-35 seconds after contrast media injection), and portal-venous-phase CT (70-80 seconds after contrast media injection). Some patients underwent additional CT angiography, with an early arterial phase (15-20 seconds after contrast media injection). The following CT findings were assessed and recorded by a radiologist specialized in abdominal imaging, with 5-years of experience in: morphologic changes of the cirrhosis, the presence of fatty liver, evidence of portal hypertension, the maximum diameter of the main portal vein, and the presence of space-occupying masses. The radiologist was blinded to the clinical data and the intraoperative findings. Fatty liver was defined by decreased attenuation of liver parenchyma, lower than that of the spleen, on non-contrast CT images. Portal venous image sets (5 $\mathrm{mm}$ thickness, axial plane) were used for the CT volumetry, from which total- and functional liver volumes were calculated. The functional liver volume was defined as the whole liver volume minus the vascular structures, bile ducts, and focal lesions. The calculation was performed on the CT workstation using a semi-automated approach (Philips Intellispace Portal CT Liver Analysis, PHILIPS) (Figure 1). For this semi-automated approach, the liver contour would be generated after the radiologist identified the relevant anatomy (intrahepatic inferior vena cava, middle hepatic vein, right hepatic vein, left portal vein bifurcation, groove for ligamentum teres). Then, the radiologist would review and refine the liver contour to ensure that the segmentation was accurate.

IBM SPSS Statistics version 21 was used for all statistical analyses. Categorial data (e.g., gender, ChildPugh classes, and the presence of liver mass) were 




Figure 1 Workstation screenshot

shown in number and percentage. Continuous data (e.g., age, portal vein diameter, and liver volume) were shown in mean \pm standard deviation (S.D.) for normally distributed data or median and interquartile range (IQR) for skewed data. The comparison of the liver volume of different groups was analyzed using non-parametric tests. The relationship between the actual liver weight and the calculated liver volume was assessed using linear logistic regression. Pearson's correlation coefficient ( $r$ ) was used to evaluate the correlation between the total- and functional liver volume; and liver volume and the resected liver weight. A formula for the calculation of the expected liver weight, based on CT liver volume, was obtained. A p-value of less than 0.050 was considered statistically significant.

\section{Results}

\section{Patient characteristics}

There were a total of 50 patients who underwent cadaveric liver transplantation during the specified period. Among these, eighteen patients were excluded, due to the following reasons: no pre-operative CT (3 patients), no record of liver weight in the operative notes (12 patients), no operative notes in the electronic medical records (3 patients). In the end thirty-two patients enrolled into the study.

Twenty-eight patients were male, and four were female. Hepatocellular carcinomas and liver cirrhosis were the two most common indications for liver transplantation; accounting for approximately $90.0 \%$. Hepatitis C viral 
infection was the commonest cause of liver cirrhosis in this study population, followed by hepatitis B viral infection. According to the Child-Pugh classification for chronic liver disease, the majority of patients were in class $\mathrm{B}$ or $\mathrm{C}$. (Table 1).

Table 1 Clinical information

\begin{tabular}{lll}
\hline Clinical data & Number & $\%$ \\
\hline Gender & & \\
Male & $28 / 32$ & 87.5 \\
Female & $4 / 32$ & 12.5 \\
Age (year) & & \\
Range & $16-63$ & \\
Median (IQR) & $51(43-54)$ & \\
Indication for liver transplantation & & \\
Hepatocellular carcinoma & $15 / 32$ & 46.9 \\
Cirrhosis & $14 / 32$ & 43.8 \\
Acute liver failure & $1 / 32$ & 3.1 \\
Budd-Chiari & $1 / 32$ & 3.1 \\
Calori's disease & $1 / 32$ & 3.1 \\
Cause of liver cirrhosis & & \\
Hepatitis C infection & $19 / 31$ & 61.3 \\
Hepatitis B infection & $7 / 31$ & 22.6 \\
Alcoholic & $1 / 31$ & 3.2 \\
Budd-Chiari & $1 / 31$ & 3.2 \\
Calori's disease & $1 / 31$ & 3.2 \\
Congenital hepatic fibrosis & $1 / 31$ & 3.2 \\
Secondary biliary cirrhosis & $1 / 31$ & 3.2 \\
Child-Pugh classification* & & \\
A & $5 / 30$ & 16.7 \\
B & $15 / 30$ & 50.0 \\
C & $10 / 30$ & 33.3 \\
\hline
\end{tabular}

*Excluding one patient with acute liver failure $I Q R=$ interquartile range

\section{Laboratory investigation}

The direct bilirubin level in Child-Pugh class C cirrhotic patients was significantly higher than in class $A$ and class $B$ ( $p$-value $=0.021$ and $p$-value $=0.043$, respectively. Similarly, the total bilirubin level in patients with Child-Pugh class C cirrhosis was significantly higher than patients with ChildPugh class A cirrhosis ( $p-v a l u e=0.044)$. The total bilirubin level in Child-Pugh class C patients was also higher than that of Child-Pugh class $B$ patients, reaching statistical significance $(p-v a l u e=0.050)$. The remaining liver function tests were not significantly different among different ChildPugh classes (Table 2).

\section{Imaging findings}

Morphologic changes of liver cirrhosis; including, nodularity of the liver surface, left lobe hypertrophy, or caudate lobe hypertrophy, were found in 31 patients $(96.9 \%)$. The modified caudate-right lobe ratios ranged from 0.49 to $1.35(0.88 \pm 0.21 \mathrm{~mm})$. There was no significant difference in the mean of the modified caudate-right lobe ratios in patients of different Child-Pugh classes. Thirty-one out of 32 patients (96.6\%) had imaging evidence of portal hypertension; including, splenomegaly, enlarged portal vein, and varices. The main portal vein diameter ranged from 11.2 to $19.1 \mathrm{~mm}(14.96 \pm 1.94 \mathrm{~mm})$. Only three patients had fatty liver based on the non-contrast CT attenuation criteria.

\section{Computed tomography volumetric assessment}

The median time needed for volume analysis was 18.38 (10.61-22.13) minutes. The calculated total liver volume ranged from $619.5 \mathrm{ml}$ to $4,924.3 \mathrm{ml}$ (median $1,255.05(980.95-1,439.75) \mathrm{ml})$, while the calculated functional liver volume ranged from $597.2 \mathrm{ml}$ to $4,837.4 \mathrm{ml}$ (median 1,210.05 (911.78-1,354.68) ml.). The total- and functional liver volumes were highly correlated with each other $(r=0.828, p$-value $<0.001)$. There was no significant difference in the liver volume among patients with- and without HCCs $(p-$ value $=0.911$ for total - and $p-$ value $=0.852$ for functional liver volume). However, the total- and functional liver volume were significantly different among cirrhosis of various etiologies $(p-$ value $=0.001$ for totaland $p$-value $=0.003$ for functional liver volume). From this pairwise comparison revealed that patients with cirrhosis of other etiologies had a significantly larger liver volume than those with hepatitis B and those with hepatitis C cirrhosis. The liver volume was not significantly different among Child- 
Table 2 Liver function test and Child-Pugh classification

\begin{tabular}{|c|c|c|c|c|c|}
\hline \multirow{2}{*}{ Liver function test } & \multicolumn{3}{|c|}{ Child-Pugh classification } & \multirow{2}{*}{ Total } & \multirow{2}{*}{ p-value } \\
\hline & $A(n=4)$ & $B(n=15)$ & $C(n=19)$ & & \\
\hline Cholesterol $^{+}$ & $123.50 \pm 33.32$ & $114.78 \pm 36.12$ & $86.56 \pm 34.63$ & $106.95 \pm 36.99$ & 0.12 \\
\hline Total protein ${ }^{+}$ & $7.27 \pm 0.61$ & $6.33 \pm 1.29$ & $6.41 \pm 1.68$ & $6.49 \pm 1.36$ & 0.47 \\
\hline Albumin $^{+}$ & $4.00 \pm 0.99$ & $3.39 \pm 0.47$ & $3.04 \pm 0.86$ & $3.27 \pm 0.81$ & 0.85 \\
\hline Globulin $^{+}$ & $3.28 \pm 1.42$ & $2.95 \pm 1.32$ & $3.57 \pm 2.18$ & $3.19 \pm 1.62$ & 0.68 \\
\hline Total bilirubin" & $1.30(0.60-1.78)$ & $1.40(0.60-3.40)$ & $4.70(2.30-24.40)$ & $2.20(1.08-5.15)$ & $0.017^{*}$ \\
\hline Direct bilirubin ${ }^{\#}$ & $0.60(0.28-0.85)$ & $0.90(0.40-2.10)$ & $2.20(1.65-17.90)$ & $1.55(0.58-2.75)$ & $0.01^{*}$ \\
\hline Alanine aminotransferase ${ }^{\#}$ & $42.00(32.25-101.25)$ & $81.00(60.00-121.00)$ & $68.00(40.50-176.00)$ & $71.0(50.75-162.25)$ & 0.153 \\
\hline Aspartate transaminase & $39.50(28.75-72.75)$ & $106.00(39.00-128.00)$ & $51.00(27.75-79.00)$ & $62.0(33.50-109.00)$ & 0.101 \\
\hline Alkaline phosphatase & $90.00(70.00-105.00)$ & $117.00(94.00-214.00)$ & $97.50(76.00-201.00)$ & $110.0(90.00-204.25)$ & 0.255 \\
\hline
\end{tabular}

'Data shown in mean \pm S.D., analysed by using one-way ANOVA with bonferoni correction

"Data shown in median (IQR), analysed by using Kruskal-Wallis test

Table 3 Estimated liver volume and the actual liver weight; according to subgroups

\begin{tabular}{|c|c|c|c|c|}
\hline \multirow{2}{*}{ Factor } & & \multicolumn{2}{|c|}{ Estimated liver volume by $\mathrm{CT}(\mathrm{ml})^{\dagger}$} & \multirow{2}{*}{ Actual weight $(\mathrm{g})^{\dagger}$} \\
\hline & & Total liver volume & Functional liver volume & \\
\hline \multicolumn{5}{|l|}{ Gender } \\
\hline \multirow[t]{3}{*}{ Male } & Min & 823.3 & 757.3 & 768.0 \\
\hline & Max & $3,821.8$ & $3,643.5$ & $3,045.0$ \\
\hline & Median (IQR) & $1,255.1(1,046.2-1,399.9)$ & $1,210.1(938.6-1,354.7)$ & $1,251.5(1,050.0-1,388.3)$ \\
\hline \multirow[t]{3}{*}{ Female } & Min & 619.5 & 597.2 & 709.0 \\
\hline & $\operatorname{Max}$ & $4,924.4$ & $4,837.5$ & $3,000.0$ \\
\hline & Median (IQR) & $2,801.3(652.2-4,906.3)$ & 1,014.0 (626.3-3,956.6) & 1,148.0 (795.3-2,560.5) \\
\hline p-value & & 1.000 & 0.680 & 0.680 \\
\hline \multicolumn{5}{|l|}{ Etiology of cirrhosis } \\
\hline \multirow[t]{3}{*}{ Hepatitis C infection } & Min & 823.3 & 797.0 & 858.0 \\
\hline & Max & $1,600.0$ & $1,511.3$ & $1,569.0$ \\
\hline & Median (IQR) & $1,284.3 \pm(1,042.6-1,347.1)$ & $1,232.9(1,012.9-1,306.0)$ & $1,220.0(1,042.0-1,345.0)$ \\
\hline \multirow[t]{3}{*}{ Hepatitis B infection } & Min & 750.4 & 713.7 & 709.0 \\
\hline & Max & $1,450.6$ & $1,392.1$ & $1,405.0$ \\
\hline & Median (IQR) & $1,057.1(927.1-1,225.8)$ & $913.8(757.3-1,187.2)$ & $1,128.0(768.0-1,264.0)$ \\
\hline \multirow[t]{3}{*}{ Other etiology } & Min & $1,587.2$ & $1,314.2$ & $1,242.0$ \\
\hline & $\operatorname{Max}$ & $4,924.4$ & $4,837.5$ & $3,046.0$ \\
\hline & Median (IQR) & $3,821.8(2,599.1-4,888.3)$ & $3,449.0(1,424.2-4,240.5)$ & $2,988.0(1,352.0-3,022.5)$ \\
\hline p-value & & $0.001^{*}$ & $0.003^{*}$ & $0.011^{*}$ \\
\hline \multicolumn{5}{|l|}{ Child-Pugh classification } \\
\hline \multirow[t]{3}{*}{ A } & Min & 939.4 & 890.3 & $1,094.0$ \\
\hline & Max & $1,587.2$ & $1,534.2$ & $1,462.0$ \\
\hline & Median (IQR) & $1,347.1(1,129.0-1,518.9)$ & $1,292.7(1,070.1-1,463.6)$ & 1,317.0 (1,178.5-1,433.0) \\
\hline \multirow[t]{3}{*}{$B$} & Min & 750.4 & 713.7 & 709.0 \\
\hline & Max & $1,600.0$ & $1,511.2$ & $1,568.0$ \\
\hline & Median (IQR) & $1,225.8(960.4-1,377.0)$ & 1,187.2 (913.8-1,334.2) & 1,182.0 (1,182.0-1,356.0) \\
\hline \multirow[t]{3}{*}{ C } & Min & 823.3 & 757.3 & 983.0 \\
\hline & Max & $4,924.3$ & $4,837.4$ & $3,000.0$ \\
\hline & Median (IQR) & $1,126.8(1,025.9-4,079.4)$ & 1,082.9 (874.0-1,896.5) & 1,241.0 (1,018.5-1,697.3) \\
\hline p-value & & 0.570 & 0.612 & 0.451 \\
\hline
\end{tabular}

${ }^{\dagger}$ Non-normal distribution data, CT=computed tomography, IQR=interquartile range 
Pugh classes and gender (Table 3). Note the discrepancy between the total- and the functional liver volume in the female subgroup. This discrepancy was caused by one particular patient who had a huge cystic liver mass, which was unroofed during the surgery. The total liver volume of this patient (which included the volume of the lesion) was much larger than the functional liver volume (which excluded the volume of the lesion) and the liver weight (the cyst was unroofed and emptied). This discordant case was labelled as the orange dot in Figure 2. There seems to be a relationship between the maximal portal vein diameter and functional liver volume, but it was not statistically significant $(p-$ value $=0.081)$.

There was only one patient with acute liver failure in our study; therefore, this patient was excluded from the regression analysis, because the data required for multivariable analysis were not available in this patient. Doing this also resulted in a more homogeneous population comprising only of patients with cirrhosis. The linear regression analysis showed a significant relationship between functional- and total liver volume and the actual resected liver weight ( $p$-value<0.001). However, compared with the total liver volume, the functional volume was more closely correlated with the actual weight of the resected liver ( $r=0.955$ vs. $r=0.786$ ) (Figure 2). Viral hepatitis also had a high negative correlation with liver weight $(r=-0.753)$. Nevertheless, multivariate analysis taking into account, the functional liver volume, the etiology-, and the severity of liver cirrhosis showed that the functional liver volume was the only independent factor significantly associated with the actual liver weight. In contrast, the etiology and the severity of liver cirrhosis did not contribute to the prediction of the actual liver weight in the presence of the functional liver volume (Table 4). We proposed the following linear regression formula to predict the expected weight of the cirrhotic liver from the functional liver volume (R-squared $=0.914)$ :

$E L W=489.531+\left(0.618^{\star} F L V\right)$

$E L W=e x p e c t e d$ liver weight, $F L V=e s t i m a t e d$ functional liver volume

Where ELW is the expected liver weight, and FLV is the functional liver volume calculated from CT.

Table 4 Correlation with actual liver weight and multivariate analysis

\begin{tabular}{|c|c|c|c|c|c|c|c|c|}
\hline \multirow{3}{*}{ Factor } & \multicolumn{2}{|c|}{$\begin{array}{c}\text { Correlation with actual } \\
\text { liver weight }\end{array}$} & \multicolumn{4}{|c|}{ Multivariate analysis } & \multicolumn{2}{|c|}{ Collinearity statistics } \\
\hline & \multirow{2}{*}{$\begin{array}{l}\text { Correlation } \\
\text { Coefficient ( } r)\end{array}$} & \multirow[t]{2}{*}{$p$-value } & \multirow{2}{*}{$\begin{array}{l}\text { Coefficients } \\
\text { (B) }\end{array}$} & \multirow{2}{*}{ p-value } & \multicolumn{2}{|c|}{$95 \%$ confidence interval } & \multirow[t]{2}{*}{ Tolerance } & \multirow[t]{2}{*}{ VIF } \\
\hline & & & & & $\begin{array}{l}\text { Lower } \\
\text { bound }\end{array}$ & $\begin{array}{l}\text { Upper } \\
\text { bound }\end{array}$ & & \\
\hline Functional liver volume & 0.955 & $<0.001$ & 0.564 & $<0.001$ & 0.44 & 0.66 & 0.486 & 2.057 \\
\hline Child-Pugh class & 0.210 & 0.265 & 3.810 & 0.930 & -84.80 & 92.42 & 0.951 & 1.051 \\
\hline $\begin{array}{l}\text { Etiology } \\
\text { (Viral hepatitis vs other) }\end{array}$ & -0.753 & $<0.001$ & -30.430 & 0.804 & -279.77 & 218.91 & 0.491 & 2.037 \\
\hline
\end{tabular}

VIF=variance inflation factor 
A


Figure 2 The relationship between CT liver volume and the actual liver weight

(A) The scatter plot shows significant linear relationship between the functional liver volume and the actual weight of the resected liver ( $\mathrm{R}$-squared $=0.914$; $p$ value $<0.001$ ).

(B) The scatter plot between the actual liver weight and the total liver volume also shows a significant linear relationship, but with a lower goodness-of-fit (R-squared=0.786, p-value $<0.001)$ 


\section{Discussion}

Computed tomography is widely recognized as an accurate modality for pre-operatively estimating liver volume. Previous studies reported a significant correlation between the predicted liver volume and the actual volume, or the actual weight of the liver. ${ }^{16,20}$ Unfortunately, most of the data were derived from healthy liver grafts of living donors. There was a study on CT volumetry in patients who underwent partial liver resection; however, this study did not take the presence of liver cirrhosis into account. ${ }^{15}$ The data on the usage of CT volumetry in diseased livers are scarce. A study by Goumard et al. showed a considerable discrepancy between the CT volume and the weight of explanted cirrhotic livers, and the liver weight, volume, and density could differ among the different etiology of cirrhosis. The study found that livers with hepatitis B virus-related cirrhosis cirrhosis had a significantly lower volume and higher density than other etiologies. The study concluded that the volume quantification by CT might not be reliable in cirrhotic patients. ${ }^{18}$

There were overlaps of liver volume and weight among patients with different severity of cirrhosis in our study, so the difference among the groups was not statistically significant. Similar to the previous study, we found that the estimated liver volume and the actual liver weight of patients with cirrhosis, due to viral hepatitis, were significantly lower than those with other etiologies. There was also a moderate negative correlation between viral-related cirrhosis and resected liver weight. However, the estimated liver volume, particularly the functional liver volume, of the diseased liver in our study was still very highly correlated with the actual liver weight $(r=0.955$, $\mathrm{p}$-value<0.001). Furthermore, multivariate analysis showed that the etiologies and disease severity did not affect the ability of CT-derived FLV in the resected liver weight prediction. One reason for the discordance between the results of both studies might lie in the difference of the study population; nearly $90.0 \%$ of patients in the current study had viral hepatitis; whereas, in the prior research viral hepatitis accounted for just above one-third of the population. In addition, even though the volume and weight of livers with viral-related cirrhosis were smaller than any other etiology, the volume and weight were still correlated, and CT could still reflect the changes accordingly.

Based on the findings in our study, from an anatomical point of view, CT is still accurate in the estimation of the volume and the weight of cirrhotic livers. However, since there was no significant relationship between the CT volume and the severity of cirrhosis, and it is common knowledge that livers with a higher degree of cirrhosis would function poorer than those with milder degrees, the application of CT volumetry on diseased livers should not be in the "one-size-fit-all" criteria. Rather, a graded surgical approach (i.e., a larger FLR should be spared for patients with a higher degree of cirrhosis, and vice versa) or additional functional assessment should be applied in this group of patients.

Typically, $1 \mathrm{~kg} \Lambda$ is generally used as the conversion factor for converting the estimated liver volume to the liver's actual weight. However, several studies suggested that this number might not be appropriate, and alternative methodsranging from a different conversion factor to a more complex formula-were proposed. ${ }^{16,19,20,25}$ Nevertheless, these suggestions came from studying normal livers and did not account for the presence of liver cirrhosis. In the current study, we proposed a formula that can accurately predict the weight of the resected cirrhotic liver $(\mathrm{R}$-squared $=0.914)$.

Liver volume estimation using CT can be classified into three approaches: manual, semi-automated, and automated. The manual approach, in which the liver contour is manually drawn by hand, is considered the most accurate; however, it is the most time-consuming method. The semi-automated approach relies on users' interaction 
and software assistance to define the liver contour. For the automated approach, the liver contour is fully calculated and outlined by the computer. The semi-automated and the automated approaches yielded comparable results to the manual measurement. ${ }^{26,27}$ In our study, we used a semiautomated approach for the volumetric analysis. This type of assessment usually takes longer than the fully automated approach. $^{27,28}$ However, the fully automated assessment is not possible in the setting of partial hepatic resection, in which the measurement needed to be tailored differently for each patient according to the surgical planning. Regarding the time needed to complete the segmentation and analysis, our median time was comparable to those reported for the same measurement approach in other studies. ${ }^{27,28}$

The limitation of this study was the small sample size, and its retrospective nature. A prospective study with a larger population is required to validate the reliability of $\mathrm{CT}$ in the pre-operative estimation of liver volume and weight of pathologic livers.

\section{Conclusion}

Computed tomography volumetric assessment strongly correlates, and can reliably predict the actual weight of the resected liver in cirrhotic patients. However, because the estimated liver volume and the actual liver weight did not correlate with the liver disease severity, a graded approach for surgery, or an additional functional assessment of the liver may be necessary for this group of patients.

\section{Funding sources}

This study was granted by the Faculty of Medicine, Khon Kaen University, Thailand (Grant Number: IN59207).

\section{Conflict of interest}

The authors declare no conflict of interest.

\section{References}

1. Chedid MF, Kruel CRP, Pinto MA, Grezzana-Filho TJM, Leipnitz I, Kruel CDP, et al. Hepatocellular carcinoma: diagnosis and operative management. Arq Bras Cir Dig 2017;30:272-8.

2. Cillo U, Fondevila C, Donadon M, Gringeri E, Mocchegiani F, Schlitt HJ, et al. Surgery for cholangiocarcinoma. Liver Int 2019; 39(Suppl 1):143-55.

3. Hadden WJ, de Reuver PR, Brown K, Mittal A, Samra JS, Hugh TJ. Resection of colorectal liver metastases and extrahepatic disease: a systematic review and proportional meta-analysis of survival outcomes. HPB 2016;18:209-20.

4. Bacalbaşa N, Balescu I, Dima S, Popescu I. Long-term survivors after liver resection for breast cancer liver metastases. Anticancer Res 2015;35:6913-7.

5. Grimme FAB, Seesing MFJ, van Hillegersberg R, van Coevorden F, de Jong KP, Nagtegaal ID, et al. Liver resection for hepatic metastases from soft tissue sarcoma: a nationwide study. DSU 2019;36:479-86.

6. Clarke NAR, Kanhere HA, Trochsler MI, Maddern GJ. Liver resection for non-colorectal non-neuroendocrine metastases. ANZ J Surg 2018;88:E313-7.

7. Gandy RC, Bergamin PA, Haghighi KS. Hepatic resection of non-colorectal non-endocrine liver metastases. ANZ J Surg 2017;87:810-4.

8. Ray S, Mehta NN, Golhar A, Nundy S. Post hepatectomy liver failure - A comprehensive review of current concepts and controversies. Ann Med Surg (Lond) 2018;34:4-10.

9. Rahnemai-Azar AA, Cloyd JM, Weber SM, Dillhoff M, Schmidt C, Winslow ER, et al. Update on liver failure following hepatic resection: strategies for prediction and avoidance of postoperative liver insufficiency. J Clin Transl Hepatol 2018;6:97104.

10. Schindl MJ, Redhead DN, Fearon KCH, Garden OJ, Wigmore SJ. The value of residual liver volume as a predictor of hepatic dysfunction and infection after major liver resection. Gut 2005;54:289-96.

11. Ribero D, Zimmitti G, Aloia TA, Shindoh J, Forchino F, Amisano $\mathrm{M}$, et al. Preoperative cholangitis and future liver remnant volume determine the risk of liver failure in patients undergoing Resection for Hilar Cholangiocarcinoma. J American Coll Surg 2016;223:87-97. 
12. Alizai $\mathrm{PH}$, Haelsig $A$, Bruners $P$, Ulmer $F$, Klink CD, Dejong $\mathrm{CHC}$, et al. Impact of liver volume and liver function on posthepatectomy liver failure after portal vein embolizationA multivariable cohort analysis. Annal Med Surg 2018;25:6-11.

13. Lim MC, Tan CH, Cai J, Zheng J, Kow AWC. CT volumetry of the liver: Where does it stand in clinical practice?. Clin Radio 2014;69:887-95.

14. Khan AS, Garcia-Aroz S, Ansari MA, Atiq SM, Senter-Zapata M, Fowler K, et al. Assessment and optimization of liver volume before major hepatic resection: current guidelines and a narrative review. Int J Surg 2018;52:74-81.

15. Karlo C, Reiner CS, Stolzmann P, Breitenstein S, Marincek B, Weishaupt D, et al. CT- and MRI-based volumetry of resected liver specimen: Comparison to intraoperative volume and weight measurements and calculation of conversion factors. European J Radiol 2010;75:e107-11.

16. Sa L, Gk B. Standard formulae in predicting liver volumes: a South East Asian Series of Adult Living Donors. J Transplant Technol Res 2016:6:1

17. Pomposelli JJ, Tongyoo A, Wald C, Pomfret EA. Variability of standard liver volume estimation versus software-assisted total liver volume measurement. Liver Transplant 2012;18:1083-92.

18. Goumard C, Perdigao F, Cazejust J, Zalinski S, Soubrane O, Scatton O. Is computed tomography volumetric assessment of the liver reliable in patients with cirrhosis?. HPB (Oxford) 2014;16:188-94

19. Zakareya T, Abbasy M, Abdel-Razek W, Abdelkader Salama I, Deif M. A novel formula for graft weight estimation from preoperative computed tomography volumetric measurement in living donor liver transplantation. Transplant Technol 2017;5:1.

20. Pinheiro RS, Cruz-Jr RJ, Andraus W, Ducatti L, Martino RB, Nacif LS, et al. Preoperative computed tomography volumetry and graft weight estimation in adult living donor liver transplantation. Abcd Arq Bras Cir Dig 2017;30:38-41.

21. Sonnemans L, Hol J, Monshouwer R, Prokop M, Klein W. Correlation between liver volumetric computed tomography results and measured liver weight: a tool for preoperative planning of Liver Transplant. Exp Clin Transplant 2016;14: 72-822.

22. Lemke A-J, Brinkmann MJ, Schott T, Niehues SM, Settmacher $U$, Neuhaus $P$, et al. Living donor right liver lobes: preoperative CT volumetric measurement for calculation of intraoperative weight and volume. Radiol 2006;240:736-42.

23. Golse N. Should we have blind faith in liver volumetry? SCR 2019: doi: 10.31487/J.SCR.2019.01.003.

24. Lo RC, Kim H. Histopathological evaluation of liver fibrosis and cirrhosis regression. Clin Mol Hepatol 2017;23:302-7.

25. Karlo C, Reiner CS, Stolzmann P, Breitenstein S, Marincek B, Weishaupt D, et al. CT- and MRI-based volumetry of resected liver specimen: Comparison to intraoperative volume and weight measurements and calculation of conversion factors. European J Radiol 2010;75:e107-11.

26. Bozkurt B, Emek E, Arikan T, Ceyhan O, Yazici P, Sahin T, et al. Liver graft volume estimation by manual volumetry and software-aided interactive volumetry: which is better? Transplant proceedings 2019;51:2387-90.

27. Suzuki K, Epstein ML, Kohlbrenner R, Garg S, Hori M, Oto A, et al. Quantitative radiology: automated CT liver volumetry compared with interactive volumetry and manual volumetry. American J Roentgenol 2011;197:W706-12.

28. Lodewick TM, Arnoldussen CWKP, Lahaye MJ, van Mierlo KMC, Neumann UP, Beets-Tan RG, et al. Fast and accurate liver volumetry prior to hepatectomy. HPB 2016;18:764-72. 